\title{
ERRATUM
}

\section{Omission-based Abstraction for Answer Set Programs - ERRATUM}

\author{
ZEYNEP G. SARIBATUR and THOMAS EITER
}

doi: 10.1017/S1471068420000095, Published online by Cambridge University Press, 09 June 2020.

The author corrections to the above mentioned article (Saribatur and Eiter 2020) were not correctly carried out with regards the equation numbers.

the labels (a)-(b)-(c) in the omit(r,A) definition at the end of page 6 should also have been changed to (o1)-(o2)-(o3) while this change was made in the text.

The publishers apologise for this error.

\section{Reference}

Saribatur, Z. And EITER, T. 2020. Omission-Based Abstraction for Answer Set Programs. Theory and Practice of Logic Programming, 1-51. doi: 10.1017/S1471068420000095 